\title{
Analysis of the phonon spectrum in the titanium oxyhalide $\mathrm{TiOBr}$
}

\author{
G. Caimi and L. Degiorgi \\ Laboratorium für Festkörperphysik, ETH Zürich, CH-8093 Zürich, Switzerland \\ P. Lemmens \\ Max Planck Institute for Solid State Research, \\ Heisenbergstr. 1, D-70569 Stuttgart, Germany \\ F.C. Chou \\ Center for Materials Science and Engineering, \\ M.I.T., Cambridge, MA 02139, U.S.A.
}

(Dated: 1st November 2018)

\begin{abstract}
We present the electrodynamic response of $\mathrm{TiOBr}$, which undergoes a transition to a spinsinglet ground state for temperatures below $\mathrm{T}_{C 1}=28 \mathrm{~K}$. The temperature evolution of the phonon anomalies indicates, like in $\mathrm{TiOCl}$, an extended fluctuation regime, extending well above $\mathrm{T}_{C 1}$. At low frequencies, the spectral weight is progressively suppressed by decreasing the temperature, suggesting the formation of a spin-gap and a considerable electron-phonon coupling. A comparison of the two oxyhalides shows a weaker interplane coupling and reduced dimensionality for $\mathrm{TiOBr}$ that enhances fluctuations and shifts the ordering temperatures to lower values. In the fluctuation regime the temperature dependencies of some phonon parameters track the temperature evolution of the magnetic susceptibility.

PACS numbers: 78.20.-e, 71.36.+c
\end{abstract}




\section{INTRODUCTION}

The research on two-dimensional (2D) high temperature superconductors raised considerable interest in related low dimensional transition metal oxides. The aim is to understand the interplay of topological aspects, strong electronic correlation and magnetism in low dimension. As quantum magnetism with spin- $\frac{1}{2}$ is characterized by strong fluctuations and suppression of long range magnetic order $\underline{\underline{1}}$ other ground states with exceptional properties may be realized. Beside $\mathrm{Cu}^{2+}$ in $3 d^{9}$ configuration with a hole in the $e_{g}$ orbitals, $\mathrm{S}=\frac{1}{2}$ quantum magnets are achieved with $\mathrm{Ti}^{3+}$ and $\mathrm{V}^{4+}$, namely in the $3 d^{1}$ configuration and one single $d$ electron occupying a $t_{2 g}$ orbital. In addition, the smaller Jahn-Teller coupling of $t_{2 g}$ with respect to $e_{g}$ orbitals allows in certain cases an additional dynamics of these orbital states. Initially, the $\mathrm{TiOX}$ compounds, with $\mathrm{X}=\mathrm{Cl}$ and $\mathrm{Br}$, were considered as $2 \mathrm{D}$ antiferromagnets and candidates for resonance valence bond ground states ${ }^{2}$. Recently, however, new experimental findings in $\mathrm{TiOCl}$ jeopardize this picture and point toward a one dimensional character of the electronic system. $\mathrm{LDA}+\mathrm{U}$ calculations predict an ordering of the $t_{2 g}$ orbitals leading to a quasi 1D antiferromagnetic spin- $\frac{1}{2}$ chain at low temperature ${ }^{3}$. Nonetheless, pronounced phonon anomalies observed in Raman and infrared (IR) spectroscopy suggest a gradual cross-over of the magnetic correlations from 1D at low temperature to $2 \mathrm{D}$ at higher temperature, due to a change of the $t_{2 g}$ admixture ${ }^{4.5}$. These experimental findings are supported by a recent band structure calculation using frozen phonons that shows a hybridization of oxygen and chlorine states and an admixture of the $d_{x z}$ and $d_{y z}$ orbitals induced by the local distortions. These latter effects can contribute to a $2 \mathrm{D}$ character of the ground state $e^{6}$.

The thorough analysis of the phonon anomalies in TiOCl (Refs. 4 and 5 ) evidenced a narrowing of the phonon modes and depression of their spectral weight $(S W)$ for temperatures below $\mathrm{T}^{*}=135 \mathrm{~K}$, which was associated with a pseudo spin-gap phase, first identified by NMR experiments ${ }^{7}$. Using this depletion of $S W$ observed in both Raman and IR spectroscopy a spin-gap of $2 \Delta \approx 430 \mathrm{~K}$ has been inferred (Refs. 4 and 5 ). The most interesting aspect of $\mathrm{TiOCl}$ is the pseudo spin-gap phase at intermediate temperature and the interplay of phonon with orbital degree of freedoms. At low temperatures, $\mathrm{T}<\mathrm{T}_{C 1}=67 \mathrm{~K}$, TiOCl shows a more conventional spin-Peierls-like (SP) behavior.

In order to broaden our knowledge on titanium oxyhalides and trying to generalize the 
picture already drawn ${ }^{4.5}$ for $\mathrm{TiOCl}$, we have investigated $\mathrm{TiOBr}$ by infrared spectroscopy. In $\mathrm{TiOBr}$, due to the larger ionic radius of $\mathrm{Br}^{-}$, a weaker interplane coupling is expected that should lead to even stronger fluctuations. In this paper we will provide a complete set of optical data, followed by a detailed analysis of the phonon spectrum of $\mathrm{TiOBr}$. A comparison with the parent compound $\mathrm{TiOCl}$ is performed as well. The manuscript is organized as follow: first we briefly describe the experimental results. We then present the analysis of the data and the related fitting procedure, which allows us to extract the relevant informations about the ground state, the spin-gap opening and the fluctuation effects in the TiOX systems.

\section{EXPERIMENT AND RESULTS}

The $\mathrm{TiOBr}$ single crystals were synthesized by vapor-transport techniques from $\mathrm{TiO}_{2}$ and $\mathrm{TiBr}_{3}$ as reported in Ref. 8. The structure of the oxyhalogenide $\mathrm{TiOBr}$ (Fig. 11) has FeOCl as archetype and is thus formed by a double layer of $\mathrm{Ti}^{3+} \mathrm{O}^{2-}$ intercalated by a $\mathrm{Br}^{-}$bilayer.

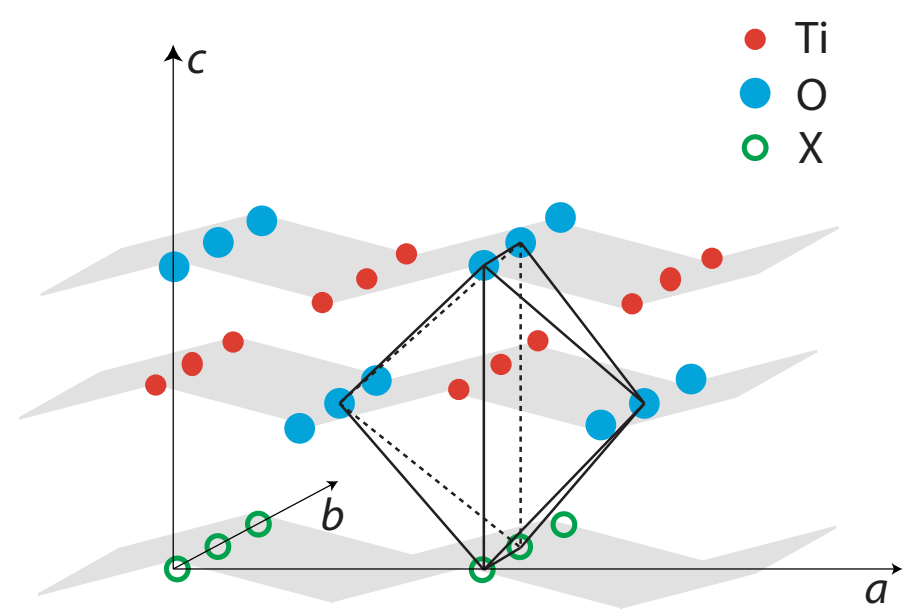

Figure 1: (Color online) Schematic representation of the crystal structure of the TiOX compounds. The octahedra surrounding each Ti ions is traced out.

The $\mathrm{Br}^{-}$bilayers mediate only weak van der Waals interactions between successive $\mathrm{Ti}^{3+} \mathrm{O}^{2-}$ bilayers, inside which $\mathrm{Ti}$ and $\mathrm{O}$ form a buckled double plane ${ }^{2}$. Each $\mathrm{Ti}$ ion is surrounded by a distorted octahedron of $\mathrm{O}$ and $\mathrm{Br}$ ions. The $\mathrm{TiO}_{4} \mathrm{Br}_{2}$ octahedra have the apexes along the $a$ axis occupied by two $\mathrm{O}$ ions. The sides parallel to the $b$ axis are formed 
either by two $\mathrm{O}$ or by two $\mathrm{Br}$ (Fig. (1). The important exchange path in the electronic structure is the direct $t_{2 g}$ orbital overlap, rather than the super-exchange via oxygen ${ }^{3}$. The $t_{2 g} d_{x y}$ orbitals form a linear chain running along the $b$ axis, linking Ti ions in the same plane $e^{\underline{3}}$. The $d_{x z}$ orbitals are rotated by $45^{\circ}$ so that two of the lobes point toward the Ti atoms of the neighbor layer, forming a zig-zag chain along the $a$ axis.

The magnetic susceptibility $\chi(\mathrm{T})$ (Ref. 9) displays a broad maximum at high temperatures for both TiOX compounds. At $\mathrm{T}_{C 1} \sim 67 \mathrm{~K}$ and $\mathrm{T}_{C 2} \sim 92 \mathrm{~K}$ a sharp drop and a kink is observed in TiOCl. In $\mathrm{TiOBr}$ these anomalies are shifted to lower temperatures, $\mathrm{T}_{C 1} \sim 28$ $\mathrm{K}$ and $\mathrm{T}_{C 2} \sim 47 \mathrm{~K}$, respectively. For $\mathrm{T}<\mathrm{T}_{C 1}$, the TiOX compounds have a non magnetic ground state, which is associated to the opening of a spin-gap and a related dimerization of the chain of $d_{x y}$ orbitals. This scenario has some similarities to a spin-Peierls transition ${ }^{3}$. The reduced $\mathrm{T}_{C}$ in $\mathrm{TiOBr}$ with respect to TiOCl may be phenomenologically explained as a consequence of the increased distance between the $\mathrm{Br}$ and $\mathrm{TiO}$ bilayers, which reduces the interplane coupling and enhances the 2D character of TiOBr. At lower dimensions, the more pronounced fluctuations hamper the formation of long-range order.

We have measured the optical reflectivity $R(\omega)$ in a broad spectral range $\left(30-10^{5} \mathrm{~cm}^{-1}\right)$ as a function of temperature ranging from 10 to $300 \mathrm{~K}$ and at selected magnetic fields 0$7 \mathrm{~T}$. As in the $\mathrm{Cl}$ compound, no magnetic field dependence is observed in $\mathrm{TiOBr}$ at any temperature. We will focus our attention on the temperature dependence only. In the far(FIR) and mid-infrared (MIR) spectral range (i.e., $30-5000 \mathrm{~cm}^{-1}$ ), the $\mathrm{R}(\omega)$ spectrum was measured with a Fourier interferometer with the sample placed inside a magneto-optical cryostat equipped with appropriate optical windows. The visible and UV spectral ranges were measured with a home made spectrometer based on a Zeiss monochromator and a commercial McPherson spectrometer, respectively. Light was linearly polarized along the chain $b$ axis and the transverse $a$ axis. The same care, as reported in Ref. 5, was applied in order to avoid leakage effects of the polarizer and to assure that no undesired projections of the light polarization along any crystallographic direction occur in the present experiment.

Figure 2 displays the temperature dependence of $R(\omega)$ in the infrared spectral range. At high frequencies the spectra are temperature independent. The upper panel shows the $\mathrm{R}(\omega)$ spectra measured with light polarized along the transverse $a$ axis, while the lower one shows the spectra taken with light polarized along the chain $b$ axis. The samples were too thin to allow an investigation of the electrodynamic response along the c-axis. Comparing 


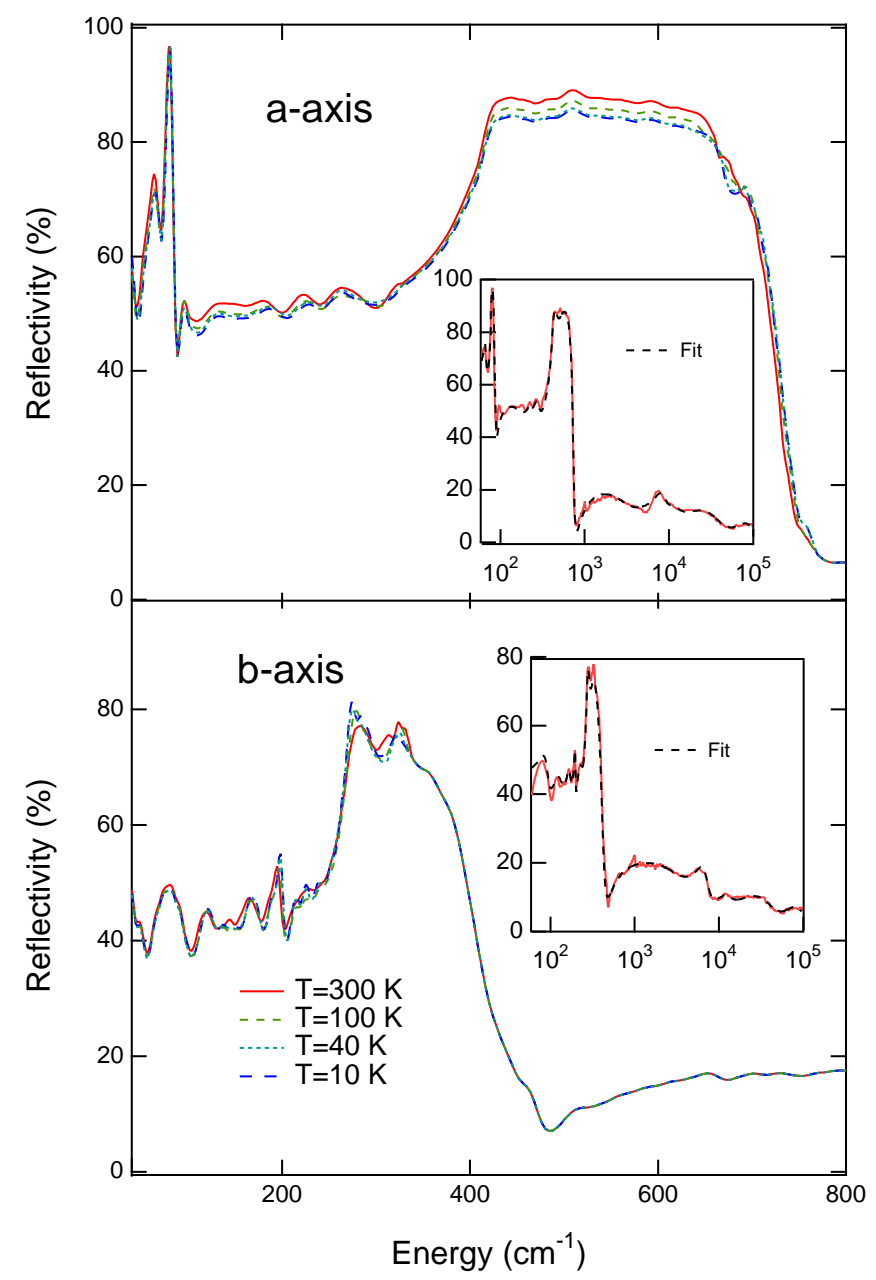

Figure 2: (Color online) $\mathrm{R}(\omega)$ of $\mathrm{TiOBr}$ at selected temperatures for light polarized along the $a$ axis (upper panel) and the $b$ axis (lower panel). The insets show the spectra in the whole measured energy interval and their Lorentz-Drude fit (see text).

both polarizations, one can appreciate the anisotropy of the reflectivity spectrum. The insets of Fig. 2 present the whole $\mathrm{R}(\omega)$ spectra up to the UV frequencies. The $\mathrm{R}(\omega)$ spectra, suitably extrapolated outside the measured frequency range (for details see Refs. 10 and 11), can be used to calculate the optical functions, such as the real part $\sigma_{1}(\omega)=\operatorname{Re}(\tilde{\sigma}(\omega))$ of the optical conductivity through Kramers-Kronig (KK) transformation. In Fig. 3 we plot the high frequency part of $\sigma_{1}(\omega)$ for both polarizations, which highlights the absorption spectrum associated with the electronic interband transitions. The FIR part of $\sigma_{1}(\omega)$ is shown in Figs. 4 and 5 . The $\sigma_{1}(\omega)$ spectra turn out to be polarization independent above 
$\sim 4 \cdot 10^{4} \mathrm{~cm}^{-1}$.

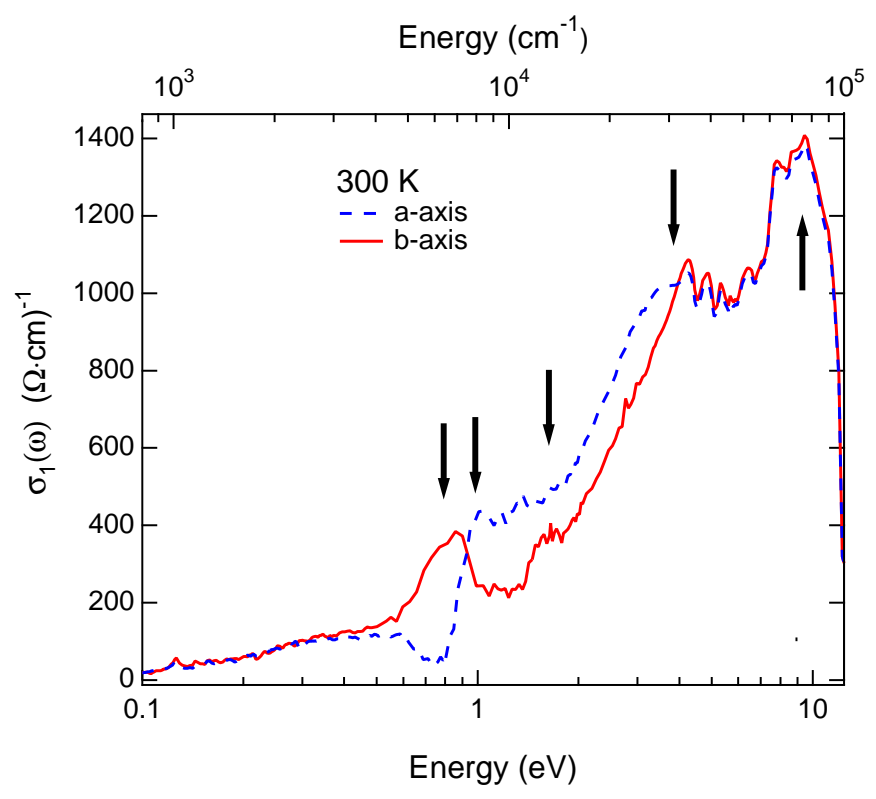

Figure 3: (Color online) High frequency part of $\sigma_{1}(\omega)$ at $300 \mathrm{~K}$ for light polarized along the $a$ and $b$ axis. The arrows indicate the characteristic absorption features (see text).

\section{DISCUSSION}

The high frequency part of $\sigma_{1}(\omega)$, reflecting the electronic interband transitions, is characterized by four main absorptions (evidenced by the arrows in Fig. 31). The first two are at $\sim 0.8-1 \mathrm{eV}$ and $\sim 1.5 \mathrm{eV}$, the third at $\sim 3.8 \mathrm{eV}$ and the fourth at $\sim 9.3 \mathrm{eV}$. These energies are comparable with those observed in TiOCl (Refs. 4 and 5 ). Recent LDA+U calculations on $\mathrm{TiOCl}^{3}$, using a full-potential LMTO method predict a split-off of the one dimensional $t_{2 g}$ bands creating an insulating charge gap of about $\sim 1 \mathrm{eV}$. This seems to occur in $\mathrm{TiOBr}$, as well. The same band structure calculation indicates interband transitions between the $\mathrm{O}$ and $\mathrm{Cl}$ p-level and the Ti d-levels at energies between 4 and $7 \mathrm{eV}$. Since the atomic distances within the $a b$ plane vary only slightly between the two compounds 2 and neglecting the interplane coupling, we can assume that the TiOX compounds have a similar electronic band structure. Therefore, it is of no surprise that both titanium oxyhalides display similar absorption features $\frac{5}{}$. 


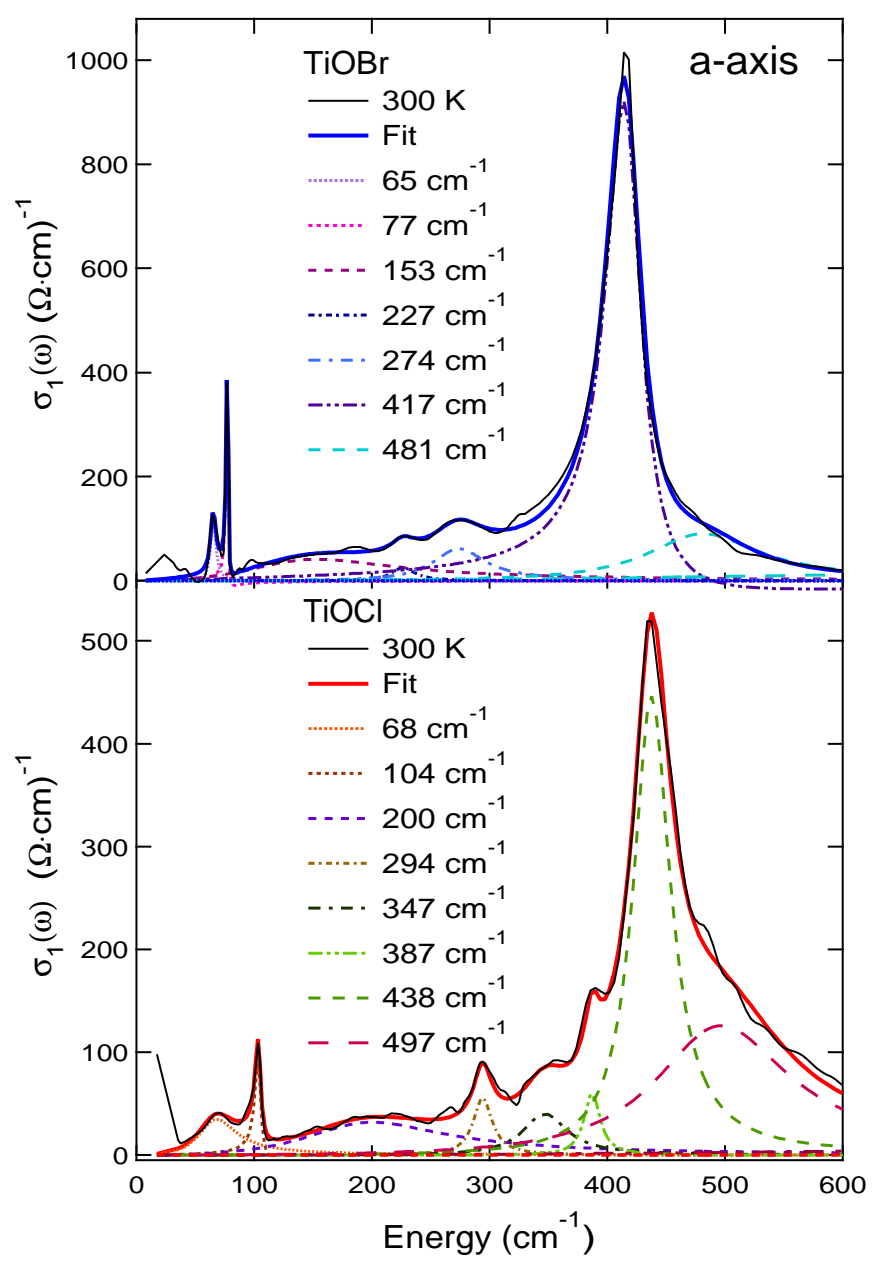

Figure 4: (Color online) The optical conductivity of TiOBr in FIR along the $a$ axis is displayed in the upper panel. The total fit and its components, identified in the legend by their respective resonance frequency, are also shown ${ }^{12}$. $\sigma_{1}(\omega)$, the total fit and its components for TiOCl (Ref. 5) are shown in the lower panel for the purpose of comparison.

Several absorptions characterize $\sigma_{1}(\omega)$ in the FIR spectral region and they are listed in Tables [1] and II for the $a$ and $b$ axis, respectively. Along the $a$ axis (Fig. 4), a strong absorption is seen at $417 \mathrm{~cm}^{-1}$, with a broad high frequency tail defining a shoulder at about $481 \mathrm{~cm}^{-1}$. At low frequencies, we detect a sharp absorption at $77 \mathrm{~cm}^{-1}$ and a small one at $65 \mathrm{~cm}^{-1}$. Along the $b$ axis (Fig. 5) a strong absorption is observed at $275 \mathrm{~cm}^{-1}$, with a shoulder at $303 \mathrm{~cm}^{-1}$ defining its high frequency tail.

In the lower panels of Figs. 4 and [5, $\sigma_{1}(\omega)$ of TiOCl has been added for the purpose 


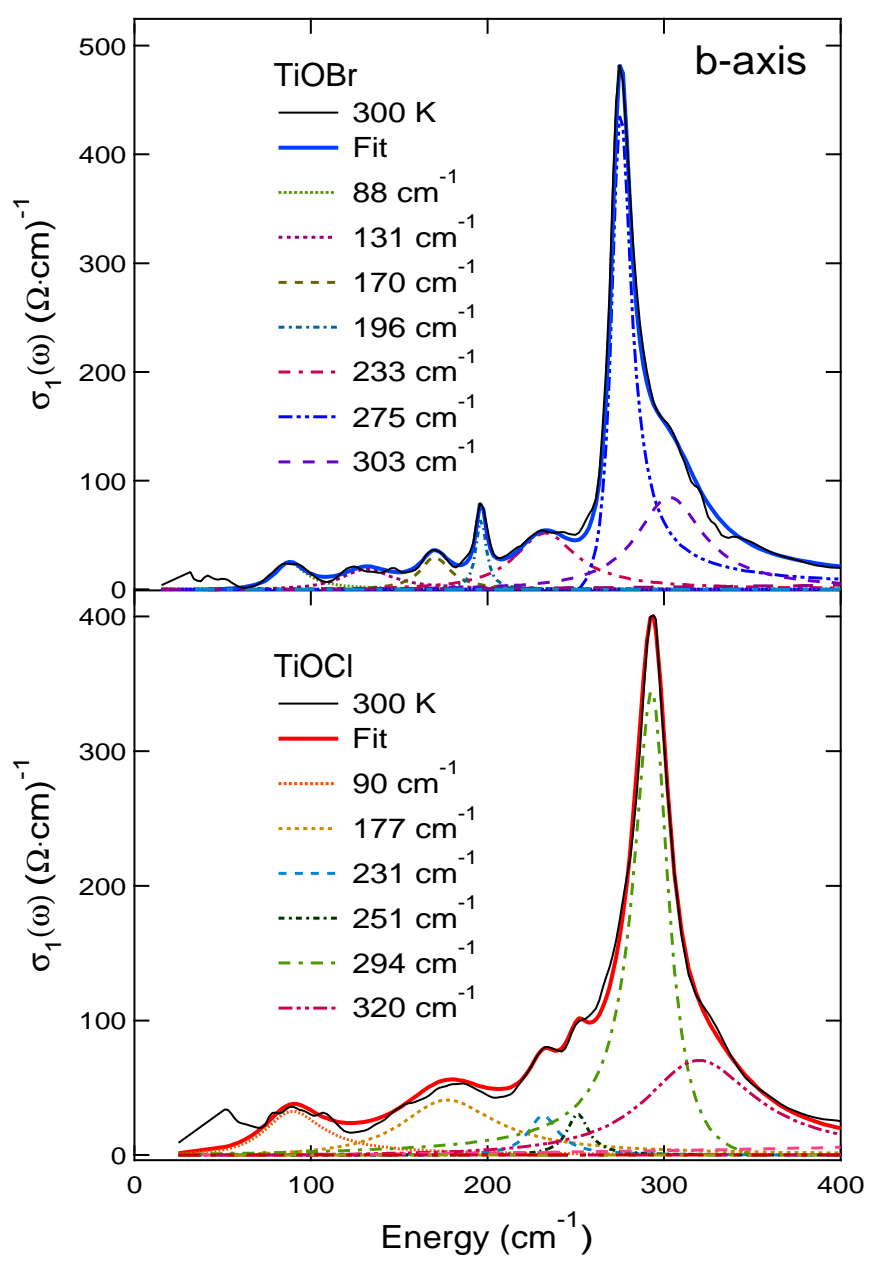

Figure 5: (Color online) The optical conductivity of TiOBr in FIR along the $b$ axis is displayed in the upper panel. The total fit and its components, identified in the legend by their respective resonance frequency, are also shown ${ }^{12}$. $\sigma_{1}(\omega)$, the total fit and its components for TiOCl (Ref. 5 ) are shown in the lower panel for the purpose of comparison.

of comparison ${ }^{5}$. One notes the overall similarity between the spectra of both compounds. Nonetheless, in TiOBr a generalized red-shift of the phonon spectrum with respect to Ti$\mathrm{OCl}$ is observed as may be expected when replacing $\mathrm{Cl}$ by a heavier element such as $\mathrm{Br}$. There are, however, some differences, like the number and shape of the absorption modes between the two TiOX compounds. For example, the strong absorption peak at $417 \mathrm{~cm}^{-1}$ in TiOBr along the $a$ axis shows a quite pronounced asymmetry, not observed at the 438 $\mathrm{cm}^{-1}$ absorption of TiOCl. Along the $b$ axis, the strong absorption at $275 \mathrm{~cm}^{-1}$ and the 
weaker modes at $233 \mathrm{~cm}^{-1}$ are well distinguished in $\mathrm{TiOBr}$, while in $\mathrm{TiOCl}$ one finds a unique asymmetric mode at $294 \mathrm{~cm}^{-1}$. Similarly, the mode at $387 \mathrm{~cm}^{-1}$ is well resolved in TiOCl but not in $\mathrm{TiOBr}$.

Table I: Resonance frequencies of the FIR absorptions in $\mathrm{TiOBr}$ (i.e., peaks in $\sigma_{1}(\omega)$ ) along the $a$ axis, determined by the Fano approach (eq. (6) ). The corresponding frequencies for TiOCl (Ref. 5) are listed for comparison. The table also reports the ratio of the resonance frequencies between the $\mathrm{Cl}$ and $\mathrm{Br}$ compound, to be compared with eq. (2) and (5). The bold frequencies refer to the $B_{3 u}$ modes.

\begin{tabular}{cccccccccc}
\hline \hline \multicolumn{1}{c}{$a$ axis } \\
\hline \hline $\mathrm{TiOBr}$ & 65 & $\mathbf{7 7}$ & 153 & 227 & 274 & $\mathbf{4 1 7}$ & 481 \\
\hline $\mathrm{TiOCl}$ & 68 & $\mathbf{1 0 4}$ & 200 & 294 & 347 & 387 & $\mathbf{4 3 8}$ & 497 \\
\hline$\omega_{0_{B r}} / \omega_{0_{C l}}$ & 0.96 & $\mathbf{0 . 7 4}$ & 0.77 & 0.77 & 0.79 & $\mathbf{0 . 9 5}$ & 0.97 \\
\hline \hline
\end{tabular}

Table II: Resonance frequencies of the FIR absorptions in $\mathrm{TiOBr}$ (i.e., peaks in $\sigma_{1}(\omega)$ ) along the $b$ axis, determined by the Fano approach (eq. (6) ). The corresponding frequencies for TiOCl (Ref. 5) are listed for comparison. The table also reports the ratio of the resonance frequencies between the $\mathrm{Cl}$ and $\mathrm{Br}$ compound, to be compared with eq. (2) and (5). The bold frequencies refer to the $B_{2 u}$ modes.

\begin{tabular}{ccccccccc}
\hline \hline \multicolumn{7}{c}{$b$ axis } \\
\hline \hline $\mathrm{TiOBr}$ & 88 & $\mathbf{1 3 1}$ & 170 & 196 & 233 & $\mathbf{2 7 5}$ & 303 \\
\hline $\mathrm{TiOCl}$ & 90 & $\mathbf{1 7 7}$ & 231 & 251 & $\mathbf{2 9 4}$ & 320 \\
\hline$\omega_{0_{B r}} / \omega_{0_{C l}}$ & 0.97 & $\mathbf{0 . 7 4}$ & 0.74 & 0.78 & $\mathbf{0 . 9 4}$ & 0.95 \\
\hline \hline
\end{tabular}

The TiOX compounds are characterized by the space group $P_{m m n}\left(59, D_{2 h}\right)$ at room temperature. The infrared active phonons as well as the eigenvectors for the atomic displacements have been established with shell model calculations. The results of this simple phonon modes calculations are reported in Ref. 5. Two $B_{3 u}$ modes polarized along the $a$ axis, two $B_{2 u}$ along the $b$ axis and two $B_{1 u}$ along the c-axis were predicted. We can describe the phonon modes of the $\mathrm{Br}$ compound using our knowledge on the $\mathrm{Cl}$ compound ${ }^{5}$ and taking 
into account the renormalization of the phonon frequencies due to the corresponding ion reduced mass and as consequence of the change in the lattice coupling, which follows from the relative volume variation of the unit cell when substituting $\mathrm{Br}$ with $\mathrm{Cl}$.

Within the linear harmonic approximation, the shift of the eigenfrequencies of the phonons is obtained by a renormalization of the oscillator strength constant $f$, when $\mathrm{Br}$ and $\mathrm{Cl}$ ions are not involved in the oscillatory displacements:

$$
\omega_{0_{B r}}=\sqrt{\frac{f_{B r}}{m}}=\sqrt{\frac{f_{C l} \cdot \frac{f_{B r}}{f_{C l}}}{m}}=\omega_{0_{C l}} \cdot \sqrt{\frac{f_{B r}}{f_{C l}}}
$$

We may estimate $\sqrt{\frac{f_{B r}}{f_{C l}}}$ for both directions ( $a$ and $b$ axis) from the IR optical active phonons, whose frequency is predicted to be independent from $m_{B r / C l}$ (Ref. 5):

$$
\sqrt{\frac{f_{B r}}{f_{C l}}}=\left\{\begin{array}{l}
\frac{\omega_{0} B r}{\omega_{0} C l}=\frac{417}{438}=0.9521 \text { for } \boldsymbol{a} \text {-axis } \\
\frac{\omega_{0_{B r}}}{\omega_{0} C l}=\frac{275}{294}=0.9354 \text { for } \boldsymbol{b} \text {-axis }
\end{array}\right.
$$

On the other hand, for the IR phonons, where the mass of the $\mathrm{Cl} / \mathrm{Br}$ ions is involved, one should consider also the renormalization due to the reduced mass.

$$
\omega_{0_{B r}}=\sqrt{\frac{f}{\mu_{B r}}}=\sqrt{\frac{f_{C l} \cdot \frac{f_{B r}}{f_{C l}}}{\mu_{C l} \cdot \frac{\mu_{B r}}{\mu_{C l}}}}=\omega_{0_{C l}} \cdot \sqrt{\frac{\mu_{C l}}{\mu_{B r}}} \cdot \sqrt{\frac{f_{B r}}{f_{C l}}}
$$

According to the eigenvector illustrated in Fig. 3 of Ref. [5, one has one atom of Ti and O for each unit cell, moving together against the $\mathrm{Br}$ atom. Therefore, the $\mathrm{Ti}+\mathrm{O}$ ensemble has a resulting atomic mass $m=m_{T i}+m_{O}=63.87 \mathrm{au}$. The reduced mass $\mu$ of the eigenmode is then:

$$
\begin{aligned}
& \mu_{C l}=\frac{m}{1+\frac{m}{m_{C l}}}=22.80 \mathrm{au} \\
& \mu_{B r}=\frac{m}{1+\frac{m}{m_{B r}}}=35.50 \mathrm{au} .
\end{aligned}
$$

for the $\mathrm{Cl}$ and $\mathrm{Br}$ compound, respectively. Inserting these reduced masses in eq. (3), the expected red-shift of the IR active phonons in TiOBr can be estimated as:

$$
\omega_{0_{B r}}=\omega_{0_{C l}} \cdot \sqrt{\frac{\mu_{C l}}{\mu_{B r}}} \cdot \sqrt{\frac{f_{B r}}{f_{C l}}}=\omega_{0_{C l}} \cdot\left\{\begin{array}{l}
0.7630 \text { for } \boldsymbol{a} \text {-axis } \\
0.7496 \text { for } \boldsymbol{b} \text {-axis. }
\end{array}\right.
$$

This simple approach accounts very well for the generalized red-shift of the phonon spectrum in $\mathrm{TiOBr}$ with respect to $\mathrm{TiOCl}$. There is an excellent agreement between the scaling 
following eq. (2) and (5) and the measured red-shift of the $B_{2 u}$ and $B_{3 u}$ modes, as may be seen by the ratios $\omega_{0_{B r}} / \omega_{0_{C l}}$ in Tables $\llbracket$ and $\amalg$. Applying this argument to the eigenfrequencies, calculated for the $B_{2 u}$ and $B_{3 u}$ modes of $\mathrm{TiOCl}$ (Ref. 5), we can anticipate the expected (theoretical) eigenfrequencies for TiOBr. These results are listed in Table III. The agreement with the experimentally determined values (Tables 【and II) is rather compelling.

Table III: IR phonon mode frequencies for $\mathrm{TiOCl}$ after the shell model calculation reported in Ref. 5 and the estimated ones for TiOBr using the renormalization factors calculated by eq. (2) and (15).

\begin{tabular}{lcccc}
\hline \hline & \multicolumn{2}{c}{$B_{2 u}(b$ axis $)$} & \multicolumn{2}{c}{$B_{3 u}(a$ axis $)$} \\
\hline TiOBr Theory & 148 & 311 & 69 & 410 \\
\hline TiOCl Theory & 198 & 333 & 91 & 431 \\
\hline \hline
\end{tabular}

Also in $\mathrm{TiOBr}$ more modes are detected than expected by symmetry ${ }^{\underline{5}}$. Nonetheless, we can extend the above analysis to these additional modes, as well. In Tables \ and 【, we have reported all mode frequencies measured along the $a$ and $b$ axis, which are compared with those of $\mathrm{TiOCl}$ (Ref. 5). It is worth noting that the modes ranging from 77 up to $347 \mathrm{~cm}^{-1}$ along the $a$ axis shift upon Br substitution of $\sim 77-79 \%$, indicating that for these modes the Br mass plays an active role. The high frequency tail of the strong phonon as well as the lowest phonon at $65 \mathrm{~cm}^{-1}$, scale by $\sim 96-97 \%$, suggesting that only the renormalization of the lattice strength plays here a relevant role. The mode scaling along the $b$ axis is similar to that of the $a$ axis. Those modes enclosed between the two predicted IR phonons $\left(131-275 \mathrm{~cm}^{-1}\right)$ show a softening of $\sim 74-78 \%$, face a prediction of $\sim 75 \%$. The shoulder at high frequency of the $B_{2 u}$ mode, generated by the displacement of the Ti and O ions, and the lowest phonon at $88 \mathrm{~cm}^{-1}$, display a shift of $\sim 95-97 \%$, confirming that in these two modes the Br displacement is generally not relevant. Even though interference effects cannot be excluded a priori, the fact that all FIR absorptions for both polarization directions scale following eq. (2) or eq. (5) supports the lattice dynamics origin for these excitations in both compounds. Similarly for $\mathrm{TiOCl}$, we believe that in $\mathrm{TiOBr}$ as well the effective space group can be used to identify a lower crystallographic symmetry. Such a lowering in symmetry enlarges the number of infrared-activated modes (like purely Raman ones), which otherwise would be silent or even IR-forbidden. 
In order to highlight the temperature dependence of the phonon spectrum, we apply the phenomenological Fano approach $\frac{13}{\underline{n}}$ to the optical conductivity $\tilde{\sigma}(\omega)$, already introduced in Ref. 5 and successfully employed for TiOCl:

$$
\tilde{\sigma}(\omega)=\sigma_{1}(\omega)+i \sigma_{2}(\omega)=\sum_{j} i \sigma_{0 j} \frac{\left(q_{j}+i\right)^{2}}{i+x(\omega)},
$$

with $x(\omega)=\left(\omega_{0 j}^{2}-\omega^{2}\right) / \Gamma_{j} \omega$, where $\omega_{0 j}$ is the resonance frequency, $\Gamma_{j}$ is the width (i.e., damping) and $\sigma_{0 j}=\omega_{p j}^{2} / \Gamma_{j} q_{j}^{2}$ with $\omega_{p j}$ as the oscillator strength and $q_{j}$ as the so-called asymmetry factor of the $j$-absorption. Eq. (6) allows us to describe the asymmetry in the line shape of the absorption features in $\sigma_{1}(\omega)$, which derives from the interaction of localized states (i.e., phonon modes) with a continuum (i.e., the continuum extends over a spectral range at energies below the localized state if $q_{j}<0$; at energies above the localized state if $\left.q_{j}>0\right)$. The fit of the complete $\sigma_{1}(\omega)$ is obtained by summing over twelve contributions (the seven shown in Figs. 4 and 5 and five more for the high frequency spectral range) for both the $a$ and $b$ axis (the complete set of fitting parameters is given in Ref. 12). The fit for the low frequency part of $\sigma_{1}(\omega)$ at $300 \mathrm{~K}$ as well as its single components are shown in Figs. 4 and 5 . The reproduction of the experimental data is astonishingly good and the same fit quality is obtained at all temperatures. The same set of fit parameters $\underline{\underline{12}}$ allows us to reproduce the measured $R(\omega)$ spectra with great fit quality as demonstrated by the insets in Fig. 2. Only the seven oscillators with the lowest energy (Figs. (4 and 5) display a temperature dependence and will be here discussed further.

The $q$ factor, describing the asymmetry of the phonon at $77 \mathrm{~cm}^{-1}$ along the $a$ axis, increases from $\sim-8$ at 300 and $150 \mathrm{~K}$ to values of about -30 at low temperatures $\underline{\underline{12}}$. This indicates a transition from an asymmetric mode to a symmetric one. Indeed, the Fano formalism for $|q|$ factors larger than 30 is almost equivalent to the (symmetric) Lorentz model. The corresponding resonance at $104 \mathrm{~cm}^{-1}$ in TiOCl is also asymmetric even though the temperature dependence of its $q$ factor is more moderate than in $\mathrm{TiOBr}$. The strong phonon at $417 \mathrm{~cm}^{-1}$ along $a$ axis, shows an asymmetric line shape, which however does not change in temperature. Along the $b$ axis, the peak at $275 \mathrm{~cm}^{-1}$ is asymmetric with constant $q$ at all temperatures. Its counterpart in $\mathrm{TiOCl}$ (i.e., the mode at $294 \mathrm{~cm}^{-1}$ ) displays a clear crossover from an asymmetric Fano-like to a Lorentzian line shape between $200 \mathrm{~K}$ and $\mathrm{T}_{C 2}$. That suggests the suppression of the continuum interacting with the phonon at $294 \mathrm{~cm}^{-1}$. Since $q<0$ the continuum extends at energies below the resonance frequency 


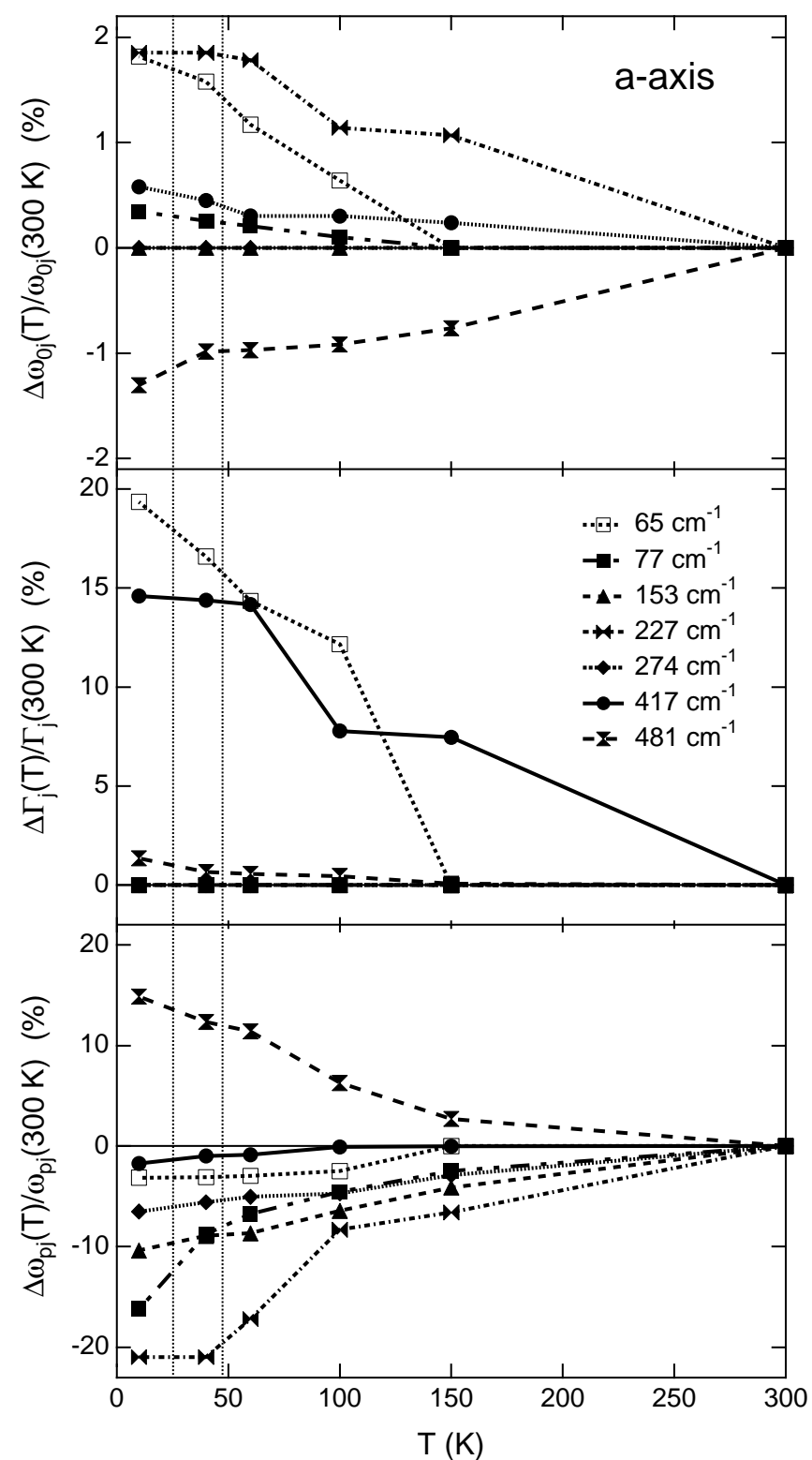

Figure 6: Temperature dependence along the $a$ axis of the percentage variation with respect to 300 $\mathrm{K}$ (see text) for the resonance frequencies $\left(\omega_{0 j}\right)$, the dampings $\left(\Gamma_{j}\right)$ and the oscillator strengths $\left(\omega_{p j}\right)$ of the phonon modes (identified in the legend by their respective resonance frequency in $\mathrm{cm}^{-1}$ ). The dotted vertical lines mark the transition temperatures $\mathrm{T}_{C 1}$ and $\mathrm{T}_{C 2}$.

of the phonon. Therefore, its suppression identifies a characteristic energy scale of $\sim 430$ K. Combining this finding with other experimental evidences, like the depletion of spectral weight at low energies 4.5 and the suppression of the Raman continuum ${ }^{4}$, the characteristic 


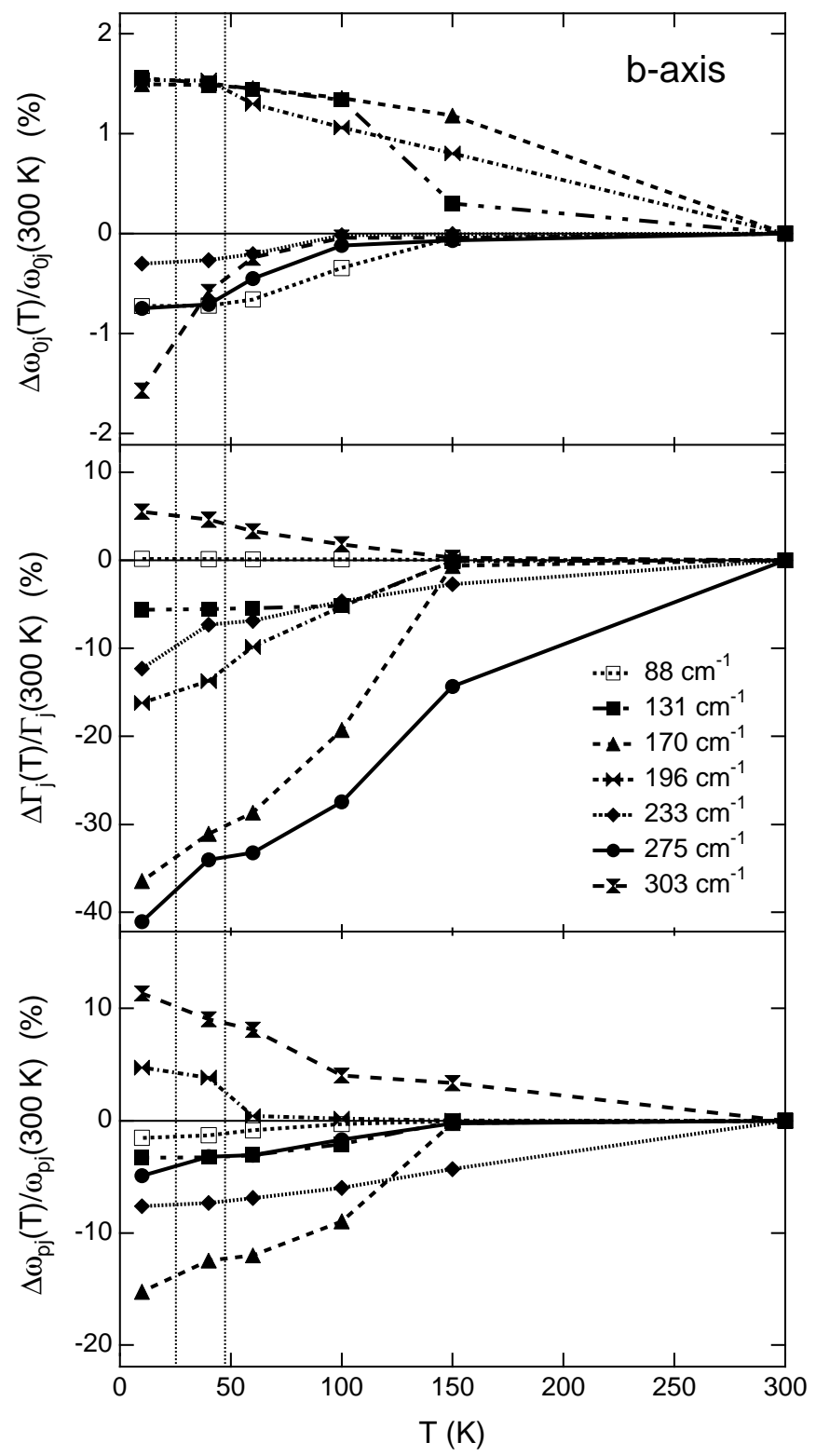

Figure 7: Temperature dependence along the $b$ axis of the percentage variation with respect to 300 $\mathrm{K}$ (see text) for the resonance frequencies $\left(\omega_{0 j}\right)$, the dampings $\left(\Gamma_{j}\right)$ and the oscillator strengths $\left(\omega_{p j}\right)$ of the phonon modes (identified in the legend by their respective resonance frequency in $\mathrm{cm}^{-1}$ ). The dotted vertical lines mark the transition temperatures $\mathrm{T}_{C 1}$ and $\mathrm{T}_{C 2}$.

energy of about $430 \mathrm{~K}$ can be associated to the opening of a spin-gap. The temperature independence of the $q$ factor for the mode at $275 \mathrm{~cm}^{-1}$ along the $b$ axis in TiOBr indicates, that the characteristic energy scale for the spin-gap lies at lower energies than $275 \mathrm{~cm}^{-1} \approx 400$ 
K.

The temperature dependence of the fit parameters ${ }^{12}\left(\omega_{0 j}, \Gamma_{j}, \omega_{p j}\right)$ is shown in Figs. 6 and 7 for both polarizations as percentage variation with respect to the $300 \mathrm{~K}$ data (e.g., $\Delta \omega_{0 j}(\mathrm{~T}) / \omega_{0 j}(300 \mathrm{~K})$, with $\left.\Delta \omega_{0 j}(\mathrm{~T})=\omega_{0 j}(\mathrm{~T})-\omega_{0 j}(300 \mathrm{~K})\right)$. The two critical temperature of the spin system, measured by $\chi(\mathrm{T})$ (Ref. 9 and 14), have been traced out with a dotted vertical line. The overall temperature dependence of the fit parameters develops in a broad temperature interval below $150 \mathrm{~K}$, and tends to saturate below $30 \mathrm{~K}$. The fact, that this temperature interval extends well above $\mathrm{T}_{C 1}$, underlines the presence of an extended fluctuation regime. Raman data confirm such a fluctuation regime in the same temperature interval14. Fluctuation effects in TiOCl have been first pointed out by NMR measurements ${ }^{7}$, where the relaxation rate of ${ }^{47,49} \mathrm{Ti}$ sites $\left(1 / \mathrm{T}_{1} \mathrm{~T}\right)$ as a function of temperature, which probes the spin degree of freedom, shows a maximum at $\mathrm{T}^{*}$. The $1 / \mathrm{T}_{1} \mathrm{~T}$ decrease below $\mathrm{T}^{*}$ indicates the suppression of low frequency spin fluctuation, and below $\mathrm{T}_{C 1}$, the relaxation rate displays an exponential decay, signaling thus the opening of a spin gap ${ }^{7}$. The temperature evolution of the relaxation rate of ${ }^{35} \mathrm{Cl}$ develops below $\sim 200 \mathrm{~K}$, suggesting that the lattice dynamic and the spin degree of freedom interplay already at high temperatures. Also electron-spin resonance (ESR) parameters highlight the strong coupling between spin and lattice degree of freedom, and they shows a progressive evolution in a temperature interval ranging from $200 \mathrm{~K}$ to $\mathrm{T}_{C 1}$ (Ref. 15).

The resonance frequency $\left(\omega_{0 j}\right)$ of almost all phonons along the $a$ axis (upper panel in Fig. (6) tends to increase with decreasing temperature, though moderately, with changes not exceeding $2 \%$. Only the highest mode at $481 \mathrm{~cm}^{-1}$ shows a weak softening. Along the $b$ axis, the low energy phonons display a weak hardening, while the three modes around the strong absorption feature peaked at $275 \mathrm{~cm}^{-1}$ show a weak softening (upper panel in Fig. (7). A softening of the phonon modes is in principal expected in models for a conventional spinPeierls transition ${ }^{16}$, where the structural deformation is driven by a linear coupling between the lattice and the magnetic degrees of freedom. However, since the dimerization must be related to normal modes away from the zone center, the softening of one or more modes across a spin-Peierls transition should be expected at the boundary of the Brillouin zone. Optical techniques can only probe the phonon branch at the $\Gamma$ point $(q=0)$. Evidence for a phonon softening at finite wave vector can therefore only be obtained by neutron scattering. Nonetheless, one can hope to gain interesting insights on the temperature dependence of the 
phonon spectrum, if the dispersion and the mixing of the branches are not too strong, so that the presence of a soft mode on the boundaries would result in an overall softening of the branch it belongs to. Thus, the weak softening of the modes at about $275 \mathrm{~cm}^{-1}$ below $100 \mathrm{~K}$ might be ascribed to a general red-shift of the $B_{2 u}$ branch. It is important to remark, that Raman scattering in $\mathrm{TiOCl}$ shows phonon modes with very large fluctuations effects and $20 \%$ softening ${ }^{4}$. The softening observed in Raman scattering, however, is of different origin, due to the different polarization of the $A_{g}$ (Raman) and $B_{2 u}$ (IR) modes involved (i.e., along $c$ and $b$ axis, respectively).

As far as the temperature dependence of the scattering rate $\left(\Gamma_{j}\right)$ is concerned (central panel in Figs. [6 and (7), along the $b$ axis almost all phonons but the one at $303 \mathrm{~cm}^{-1}$ narrow with decreasing temperature. As in $\mathrm{TiOCl}$, we associate this narrowing of the phonons along the $b$ axis to the suppression of low frequency spin fluctuations. This narrowing is detected in phonons peaked at lower frequencies, suggesting that the spin-fluctuation develops in $\mathrm{TiOBr}$ at lower energies. On the contrary, the modes at 417 and $481 \mathrm{~cm}^{-1}$ along the $a$ axis show a broadening with decreasing temperature, while in $\mathrm{TiOCl}$ the corresponding modes narrow at low temperature. The remaining modes along $a$ axis do not change the width with temperature (Fig. 6).

The mode strength $\omega_{p j}$ for the great majority of phonons decreases at low temperature for both polarizations. Only the strength of the phonons at $481 \mathrm{~cm}^{-1}$ for the $a$ axis and at $303 \mathrm{~cm}^{-1}$ and $196 \mathrm{~cm}^{-1}$ (though moderately) for the $b$ axis increases. The spectral weight $(S W)$, defined by

$$
S W=\frac{\sum_{j} w_{p j}^{2}}{8}=\int \sigma_{1}(\omega) d \omega,
$$

displays an overall depletion with decreasing temperature at frequencies below the strong modes at 417 and $275 \mathrm{~cm}^{-1}$ for the $a$ and $b$ axis, respectively. Like in TiOCl, the suppressed spectral weight is redistributed to higher frequencies. This is shown in Fig. 8, which visualizes the temperature dependence of the spectral weight redistribution. The decreasing $S W$ is obtained by summing the squared oscillator strength of each mode at energies smaller than 417 or $275 \mathrm{~cm}^{-1}$ for the $a$ and $b$ axis, respectively. The increasing $S W$ is encountered in the high frequency tail of the strong IR phonons. An equivalent analysis (eq. (7)) may be performed by integrating $\sigma_{1}(\omega)$ either from 0 to an appropriate cut-off energy or from such a cut-off energy up to energies where the $\sigma_{1}(\omega)$ spectra at different temperatures are 


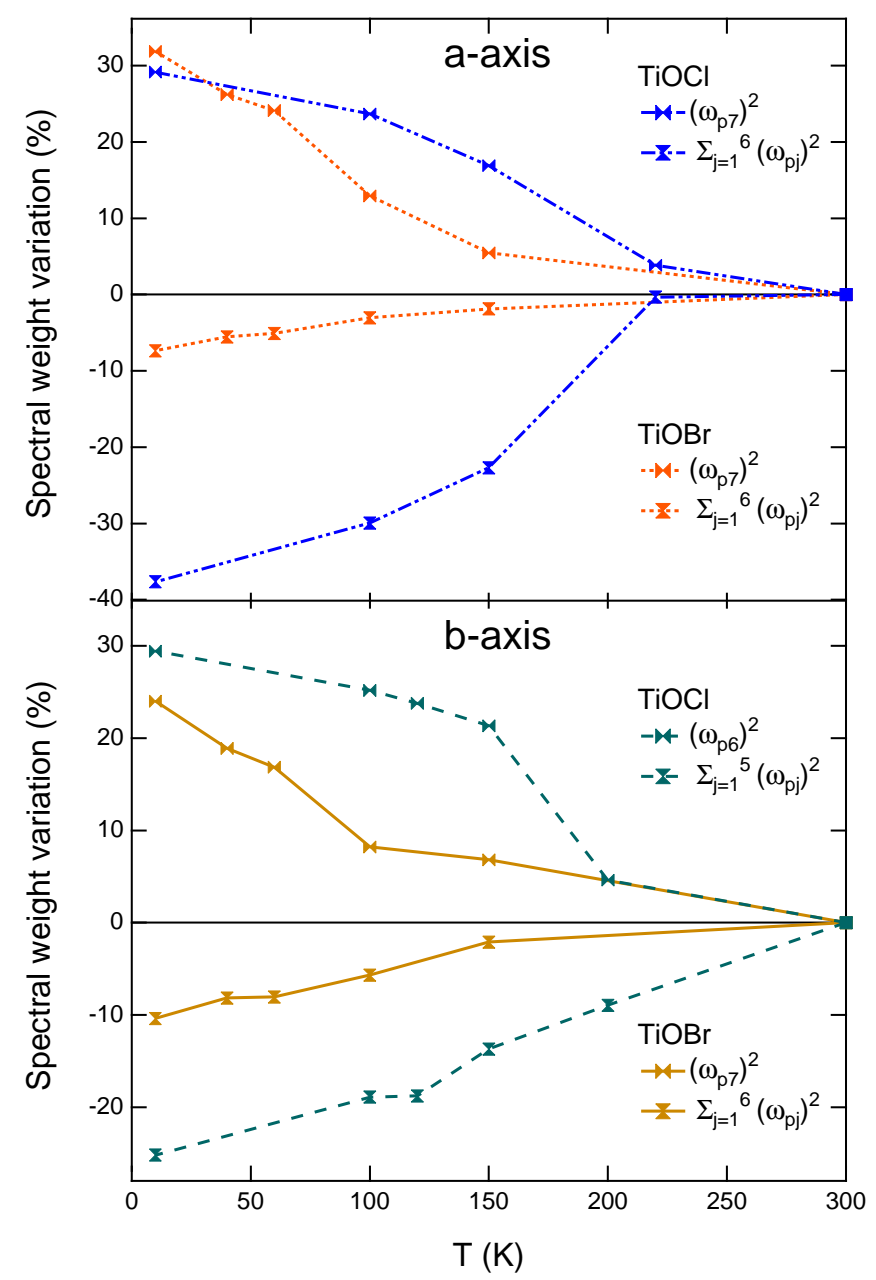

Figure 8: (Color online) Temperature dependence of the spectral weight variation for both polarizations and both samples. The figure highlights the redistribution of spectral weight between low (decreasing $S W$ with decreasing temperature) and high (increasing $S W$ with decreasing temperature) frequency (see text).

no more distinguishable. The cut-off energy can be chosen in such a way to differentiate between energy intervals where a depletion, respectively a gain in spectral weight has been established (i.e., 417 and $275 \mathrm{~cm}^{-1}$ for the $a$ and $b$ axis, respectively). Both analyses show that the total spectral weight is fully conserved at all temperatures from $\sim 1000 \mathrm{~cm}^{-1}$ on.

In $\mathrm{TiOCl}$, IR optical and Raman spectroscopy ${ }^{4.5}$ displayed a progressive suppression of $S W$ by decreasing temperature below $200 \mathrm{~K}$. The energy interval, where the $S W$ depletion occurs in $\mathrm{TiOCl}$, defines a characteristic energy scale, attributed to the spin-gap energy 
$2 \Delta_{\text {spin }} \approx 430 \mathrm{~K}$. The already stressed similarity in the two TiOX compounds leads us to associate by analogy the depletion of $S W$ in $\mathrm{TiOBr}$, although less pronounced than in $\mathrm{TiOCl}$, to the opening of the spin-gap. The softer onset of the spectral weight depletion (compared to $\mathrm{TiOCl}$ ) might indicate the reduced role of lattice dynamics in driving the magnetic phase transition at $\mathrm{T}_{C 1}$. The variation of $S W$ in TiOBr happens at temperatures $(100-150 \mathrm{~K})$ extending well above $\mathrm{T}_{C 1}$ and $\mathrm{T}_{C 2}$, pointing out again the importance of fluctuation effects. Comparing the temperature evolution of $S W$ in $\mathrm{TiOBr}$ with that of $\mathrm{TiOCl}$, one notes that the redistribution of $S W$ in $\mathrm{TiOBr}$ develops at lower temperature than in $\mathrm{TiOCl}$. This goes hand in hand with the temperature dependence of $\chi(\mathrm{T})$, signaling lower critical temperature for the spin-gap phase.

\section{CONCLUSION}

We have provided a complete analysis of the IR properties of $\mathrm{TiOBr}$, with particular attention on the phonon spectrum. We have shown that mass and oscillator strength renormalization govern the softening of the phonon modes of $\mathrm{TiOBr}$ with respect to those of $\mathrm{TiOCl}$. The temperature dependence of all relevant parameters determining the IR absorption spectrum develops over a broad temperature interval extending far above $\mathrm{T}_{C 1}$. As for $\mathrm{TiOCl}$, there is an extended fluctuation regime in $\mathrm{TiOBr}$ too. The suppression of spectral weight at low frequency by decreasing temperature hints furthermore to the opening of a spin-gap. Moreover, the temperature dependence of the $S W$ redistribution mimics the behavior of the spin susceptibility for both compounds, implying a spin-gap phase at lower temperatures in $\mathrm{TiOBr}$ than $\mathrm{TiOCl}$. In the TiOX systems the transition at $\mathrm{T}_{C 1}$ is not a conventional spin-Peierls one. As pointed out for $\mathrm{TiOCl}$, the large phonon anomalies ${ }^{4}$, the large g-factor line-width $\frac{15}{5}$, as well as the presence of an intermediate phase between $\mathrm{T}_{C 2}$ and $\mathrm{T}_{C 1}$ (Ref. 7) hint to competing lattice, spin and orbital degrees of freedom as the driving force for the transition.

\section{Acknowledgments}

The authors wish to thank J. Müller for technical help, and B. Schlein and A. Perucchi for fruitful discussions. This work has been supported by the Swiss National Foundation for 
the Scientific Research, INTAS 01-278, DFG SPP1073 and by the MRSEC Program of the National Science Foundation under award number DMR 02-13282.

1 P. Lemmens, G. Günterodt, and C. Gros, Phys. Rep. 375, 1 (2003).

2 R. J. Beynon and J. A. Wilson, J. Phys.: Condens. Matter 5, 1983 (1993).

3 A. Seidel, C. A. Marianetti, F. C. Chou, G. Ceder, and P. A. Lee, Phys. Rev. B 67, 020405 (2003).

4 P. Lemmens, K. Y. Choi, G. Caimi, L. Degiorgi, N. N. Kovaleva, A. Seidel, and F. C. Chou, cond-mat/0307502.

5 G. Caimi, L. Degiorgi, N. N. Kovaleva, P. Lemmens, and F. C. Chou, Phys. Rev. B 69, 125108 (2004).

6 T. Saha-Dasgupta, R. Valenti, H. Rosner, and C. Gros, cond-mat/0312156.

7 T. Imai and F. C. Chou, cond-mat/0301425.

8 H. Schaefer, F. Wartenpfhul, and E. Weise, Z. Anorg. Allg. Chem. 295, 268 (1958).

9 F. C. Chou, private comunication.

10 F. Wooten, Optical Properties of Solids (Academic Press, New York, 1972).

11 M. Dressel and G. Grüner, Electrodynamics of Solids (Cambridge University Press, Cambridge, 2002).

12 The complete set of fit parameters for all temperatures can be found at the link: http://www.solidphys.ethz.ch/spectro/suppinfo/TiOBr.pdf.

13 U. Fano, Phys. Rev. 124, 1866 (1961).

14 G. Caimi, L. Degiorgi, and P. Lemmens, in preparation.

15 V. Kataev, J. Baier, A. Möller, L. Jongen, G. Meyer, and A. Freimuth, Phys. Rev. B 68, 140405 (2003).

16 M. C. Cross and D. S. Fisher, Phys. Rev. B 19, 402 (1979). 